\title{
Nobiletin Enhances the Chemotherapeutic Efficacy of Docetaxel through Inhibition of Phosphatidylinositol 3-Kinase/Protein Kinase B and Induction of Apoptosis in Colon Cancer Cells
}

\begin{abstract}
C. LI, CHUNLING ZHANG ${ }^{1}$, YANFEI WANG ${ }^{2 *}$, ALAA YOUSEF GHIDAN ${ }^{3}$ AND T. M. ANTARY4
Department of Anorectal, Affiliated Hospital of Taishan Medical University, ${ }^{1}$ Department of emergency, Taian City Central Hospital, Taian, Shandong Province 271000, China, ${ }^{2}$ Department of General Surgery, The Second People Hospital of Dezhou, Dezhou, Shandong Province 253000, China, ${ }^{3}$ Nanotechnology and Microbiology Research and Development Centre, The Higher Council for Science and Technology, Amman 11941, Jordan, ${ }^{4}$ Faculty of Agriculture, The University of Jordan, Amman 11942, Jordan
\end{abstract}

\section{Li et al:: Nobiletin Combined Docetaxel Induces Apoptosis in Colon Cancer}

\begin{abstract}
Nobiletin is considered a phyto-flavonoid extracted from citrus peel and shown to several pharmacological roles such as anti-inflammatory, anti-tumor and neuroprotective properties. In this study, we investigate the role of nobiletin combined docetaxel enhances apoptosis in colorectal cancer cell lines. Our results confirmed nobiletin enhanced docetaxel-induced reactive oxygen species generation of human colorectal carcinoma cells and also enhances mitochondrial membrane potential damage in human colorectal carcinoma cells. We have also been noticed that nobiletin improved docetaxel mediated cell death confirmed by dual acridine orange/ethidium bromide assay. Phosphatidylinositol 3-kinase/protein kinase B phosphorylation heavily involved in cancer cell survival and proliferation. In this study, nobiletin inhibits phosphatidylinositol 3-kinase/protein kinase B's phosphorylation when combined with docetaxel treated human colorectal carcinoma cells. Moreover, nobiletin with docetaxel augmented the expression levels of pro-apoptotic genes such as Bax, cytochrome c, caspase 9 and caspase 3 proteins and foremost down regulation of anti-apoptotic gene protein B-cell lymphoma 2 . Hence, we confirmed that nobiletin possibly augmenting colon cancer cells compassion to docetaxel chemotherapy and offer a cellular origin for nobiletin's latent tenders in the chemo sensitization of human colorectal carcinoma cells.
\end{abstract}

Key words: Nobiletin, docetaxel, colon cancer, human colorectal carcinoma cell line, apoptosis

Colon cancer, an uncharacteristic cellular growth in the large intestine, which can be initiate from mutually inherited as well as somatic gene mutations that persist more in the course of a lifetime, even as Colorectal Cancer (CRC) resultant from an accumulation of epigenetic and genetic variations in the epithelial cells of colon region, which in turn transmute into adenocarcinoma $^{[1,2]}$. The diagnostic range of CRC is as the third grade position in males and second position in females and the 5 y survival rate is comparatively low than that of other cancers. In Saudi Arabia, 52.4 $\%$ of males and $47.6 \%$ of females were affected by $\mathrm{CRC}$ with the median age of 60 and $55 \mathrm{y}$ respectively ${ }^{[3]}$. Cancer Multidrug Resistance (CMDR) is a foremost factor affecting chemotherapy efficiency, which may eventually lead to chemotherapy dissatisfaction ${ }^{[4]}$. An enormous quantity of fundamental mechanisms

*Address for correspondence

E-mail: lichengzhen@sina.com

September-October 2021 conferring resistance towards drug has been depicted in the earlier period decades. It has been primarily divided addicted to two main categories includes noncellular and the mechanism of cellular resistance. Noncellular mechanism refers to the extracellular factors, including restricted vascular accessibility and tumor micro environment ${ }^{[5]}$.

Combination therapy permits targeting concurrently various pathways implicated in cancer, taking advantage of diverse mechanisms of action to weaken tumor drug

This is an open access article distributed under the terms of the Creative Commons Attribution-NonCommercial-ShareAlike 3.0 License, which allows others to remix, tweak, and build upon the work non-commercially, as long as the author is credited and the new creations are licensed under the identical terms

Accepted 08 October 2021

Revised 11 February 2021

Received 12 March 2020

Indian J Pharm Sci 2021;83(5):1057-1064 
resistance ${ }^{[5]}$. The Phosphatidylinositol 3-Kinase/Protein Kinase B (PI3K/AKT) concern as a cell survival pathway represents an essential target for chemotherapy mediated apoptosis. The PI3K pathway employs its roles to regulate various biochemical functions, including cell transformation, tumor growth, cell proliferation, apoptosis and angiogenesis ${ }^{[6]}$. Stimulation of apoptosis mediated cell death is the wide ranging mechanism by which nearly all therapeutic drugs eradicate tumor cells. Though, evading of apoptosis might contribute to tumor growth, development, progression and drug resistance. As apoptosis repressing the drug-resistant cancer cells, reinstatement of apoptotic signaling pathway and the highest level of cell growth hangup via substitute pathways in response to cell death is planned to be a playful way to treat drug resistant cancer cells $s^{[7,8]}$.

Docetaxel, a semi-synthetic toxoid fashioned from the unprovoked of the Taxus baccata tree and this drug has considered as most remarkable novel and dynamic chemotherapeutics in the market ${ }^{[9]}$. It has potential action towards the treatment of unruly human solid tumors, including colon cancers. Though docetaxel acquires latent cell slaughter action in a diversity of tumor cells, the high dose use of this agent has revealed to provoke diverse stages of toxic reactions, on the other hand, low as well as a moderate level of docetaxel doses, does not illustrate significant anti-tumor action in patients ${ }^{[10]}$. Consequently, the docetaxel most probably used as a monotherapy against numerous cancers has been precluded, instead of usage of combination therapy by more than one anti-cancer drug to achieve the finest possible outcome against cancer therapy ${ }^{[5,11]}$.

Nobiletin is a polymethyl attached flavonoid extracted from the peels of Citrus reticulata Blanco. It has been accounting to exhibits a vast range of biological properties like anti-cancer, anti-inflammation and neuroprotective properties. It exerts its anti-cancer properties in various cancers by restraining cell proliferation and angiogenesis and probably leading to apoptotic cell death $^{[12,13]}$. Nevertheless, the beneficial therapeutic action of computational nobiletin and chemotherapeutical drugs on cancer has not been reported. Particularly in Colon cancer, drug resistance is still a barrier to flourishing new therapeutic approaches are desired instantaneously ${ }^{[14]}$. Therefore, research investigation towards the fundamental mechanisms conferring drug resistance and the improvement of secure and efficient reverse agents by targeting these mechanisms would be a promising role in the prosperous chemotherapy in opposition to colon cancer. In this investigation, we have studied nobiletin combined docetaxel mediated cancer cell death in Human Colorectal Carcinoma Cell Line (HCT-116 cell lines).

\section{MATERIALS AND METHODS}

\section{Reagents and chemicals:}

Nobiletin, docetaxel and 3-(4,5-dimethyl-2-thiazolyl)2,5-diphenyltetrazolium bromide (MTT) were obtained from the company Sigma. Chemicals for cell culture includes solution of penicillin-streptomycin, Fetal Bovine Serum (FBS), Dulbecco's Modified Eagle Medium (DMEM) medium, Ethylene Diamine Tetraacetic Acid (EDTA), glutamine, Phosphate Buffered Saline (PBS), glutathione, trypsin and Caspase-3 and 9 was obtained from cell signaling technology. The antibodies for Phosphoinositide 3-Kinase (PI3K), Protein kinase B (AKT), Tumor protein P53 (p53), cytochrome-c, B-cell lymphoma 2 (BCL-2) and BCL2 Associated X, Apoptosis Regulator (BAX) were bought from Santa Cruz Biotechnology, China.

\section{Cell culture:}

HCT-116 colon cancer cell lines were gained from the American type of culture collection and were cultured in the occurrence of DMEM with FBS (10\%) and penicillin/streptomycin (1\%). The cell lines were stored in $5 \% \mathrm{CO}_{2}$ incubator with maintaining $37^{\circ}$ temperatures regularly.

\section{MTT assay:}

The cytotoxicity result of nobiletin and docetaxel was deliberate by MTT based colorimetric assay ${ }^{[15]}$. HCT-116 cells were equally seeded in 96-well plates (5000-6000 cells/each well) and kept incubation for $24 \mathrm{~h}$ at $37^{\circ}$ and then cells have been treated with rang of concentrations of nobiletin allow keep incubation for $24 \mathrm{~h}$ at $37^{\circ}$. After that, the cells were kept incubation with docetaxel and nobiletin (different concentrations) were added into selected wells. Later than $24 \mathrm{~h}$ incubation (at $\left.37^{\circ}\right)$, MTT solution (100 $\mu \mathrm{l}$ from $\left.5 \mathrm{mg} / \mathrm{ml}\right)$ was added to all the wells and the plates were kept $4 \mathrm{~h}$ incubation at $37^{\circ}$. Dimethylsulfoxide (DMSO) $(100-200 \mu \mathrm{l})$ was then added in all the wells for dislodging the crystal formazan. Finally, the absorbance was measured via microplate reader at $570 \mathrm{~nm}$. 


\section{2'-7'dichlorofluorescin staining for Reactive Oxygen Species (ROS) detection:}

Intra-cellular ROS was evaluated by means of DCFHDA (non-fluorescent probe) which can liberally pierce into intra-cellular matrix wherever been oxidized into fluorescent Dichlorofluorescein (DCF) by $\mathrm{ROS}^{[16]}$. As a result, the fluorescence green intensity is straightly relatively into the quantity of ROS production. HCT116 cells were plated in 6 numbered well plates and treated with nobiletin and docetaxel, then kept for incubation by using $\mathrm{CO}_{2}$ incubator for $24 \mathrm{~h}$. Later, the cells were stained with DCFH-DA for 10 min. Finally, the intensity was measured by the help of Tecan Multimode reader.

\section{Rhodamine 123 (Rh-123) staining:}

Rh-123 and a lipophilic based cationic probe were used to detect the mitochondrion membrane alteration. The cells were placed in 6 numbered plates and allowed the proper growth of cells and then it was treated with nobiletin and docetaxel. After the $24 \mathrm{~h}$, cells were incubated for 30 min with Rh-123 dye. Finally, the fluorescent intensity was measured by the help of Tecan Multimode reader.

\section{Fluorescence microscopic examination for cell death:}

The Acridine Orange (AO)/Ethidium Bromide (EB) twofold staining assay was employed for the detection of apoptosis via morphological examination ${ }^{[17]}$. The cells were treated with nobiletin and docetaxel for $24 \mathrm{~h}$. Then, the dye mixture $(20 \mu 1)$ was supplemented to the treated cells and instantaneously cells were visualized using a fluorescent microscope.

\section{Western blot:}

Cells were exposed with nobiletin and docetaxel and then protein was extracted with cell lysed Radio Immuno Precipitation Assay buffer (RIPA buffer) with addition of protease cocktail inhibitor. After that, the supernatant was used as the protein and the concentration was quantitatively estimated by standard Lowry method ${ }^{[18]}$. Each sample with $30 \mu \mathrm{g}$ of proteins was performed to the separation occur on the 8-12 \% Sodium Dodecyl Sulphate Poly Acrylamide Gel Electrophoresis (SDS-PAGE) constantly started by 70 volts. After the separation, proteins were gently transferred into nitrocellulose membrane $(0.45 \mathrm{~mm})$. The membrane was then allowed to soak on the blocking buffer for $1 \mathrm{~h}$ using bovine serum albumin (5\%). Then, appropriate primary antibodies are diluted as per the company instruction and membrane was allowed to treat primary antibodies at least $6 \mathrm{~h}$. Then the antibody treated membrane was rinsed gently with Tris Buffered Saline+Tween 20 (TBST) for 3 times. Following, the membrane again treats with the addition of 20 antibodies for $1 \mathrm{~h}$. Then it was washed three times by using TBST and chemiluminescent substrate was used to detect the bands.

\section{Statistical Examination:}

In this study, mean \pm standard deviation was used for the obtained results and there are three separated experiments were done. Besides, substantial variances between the control HCT-116 and nobiletin with docetaxel treated HCT-116 were statistically proved by Duncan's Multiple Range Test (DMRT) using with SPSS 11.0 version software package. The value $p<0.05$ was considered as statistically significant.

\section{RESULTS AND DISCUSSION}

To expand the anti-cancer activity of nobiletin, HCT116 cells were treated with nobiletin at different concentrations. Fig. 1A exhibits nobiletin considerably decreased the HCT-116 cell viability in a concentration reliant manner. Further, the proliferation of HCT-116 cells have been noticeably decreased after docetaxel exposure and the cytotoxic effect was more improved when combined with nobiletin (fig. 1B). These results advocate that the nobiletin and docetaxel act might synergistically develop toxicity in HCT-116 cell lines.

The estimation of ROS production was predictable by using DCFH-DA staining. Fig. 2 illustrated the intensity of ROS in nobiletin and docetaxel treated cells. HCT116 cells were patently treated with the incorporation of nobiletin and docetaxel showed noticeably enhanced ROS production level, which demonstrates excessive green coloured fluorescence compared to nobiletin docetaxel only treated cells.

The early apoptotic phase is activated through the amendment of Mitochondrial Membrane Potential loss (MMP) was evaluated by using lipophilic cationic dye and $\mathrm{Rh}-123$. Control cells produce an elevated intensity of green colored fluorescence representing polarized mitochondrialmembranethan the nobiletinand docetaxel alone treated cells (fig. 3). Conversely, nobiletin and docetaxel combined treatment demonstrated significant alteration of Dilated cardiomyopathy (DCM) and shows reduce green fluorescence in HCT-116 cells than 
A)

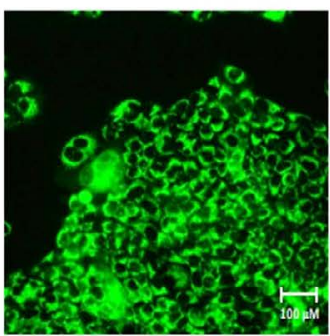

Control

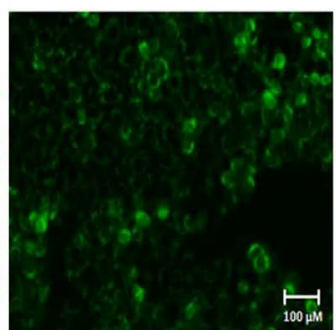

Nobiletin $(50 \mu \mathrm{M})$

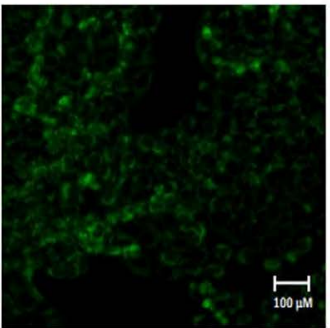

Docetaxel (100 nM)

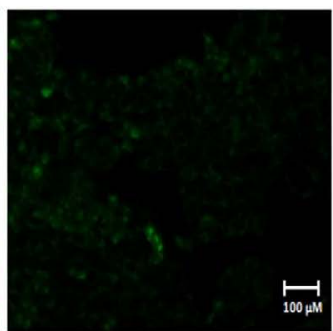

Nobiletin $(50 \mu \mathrm{M})+$ Docetaxel (100 nM)

B)

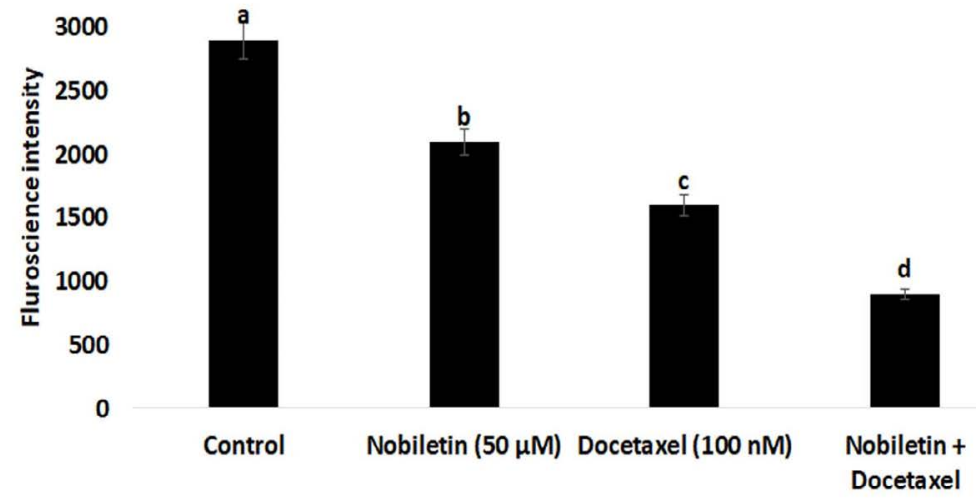

Fig. 1: The cytotoxic effect of nobiletin against colon cancer cells HCT-116; (A) cytotoxicity effect of nobiletin against HCT-116 cells measured by MTT assay; (B) The toxic effect nobiletin with different concentration docetaxel in HCT-116 cells were measured by MTT assay. The statistical analysis was carried out using one way analysis of variance. Values are represented mean \pm standard deviation of three experiments. $\mathbf{p}<0.05$ was significantly different from the control sample.

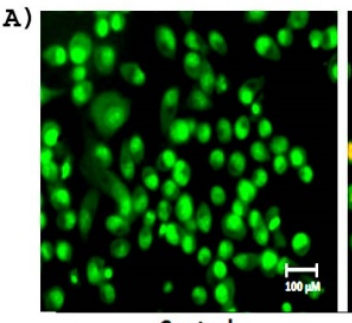

Control

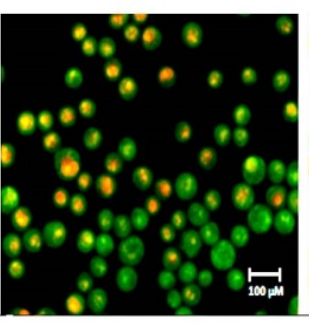

Nobiletin $(50 \mu \mathrm{M})$

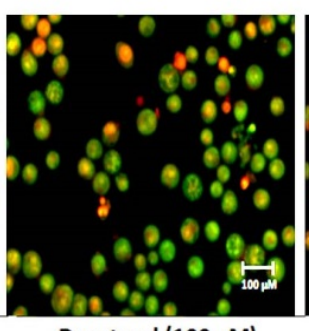

Docetaxel $(100 \mathrm{nM})$

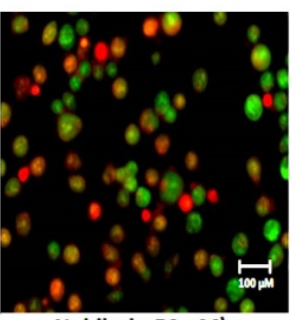

Nobiletin $50 \mu \mathrm{M})+$ Docetaxel $(100 \mathrm{nM})$

B)

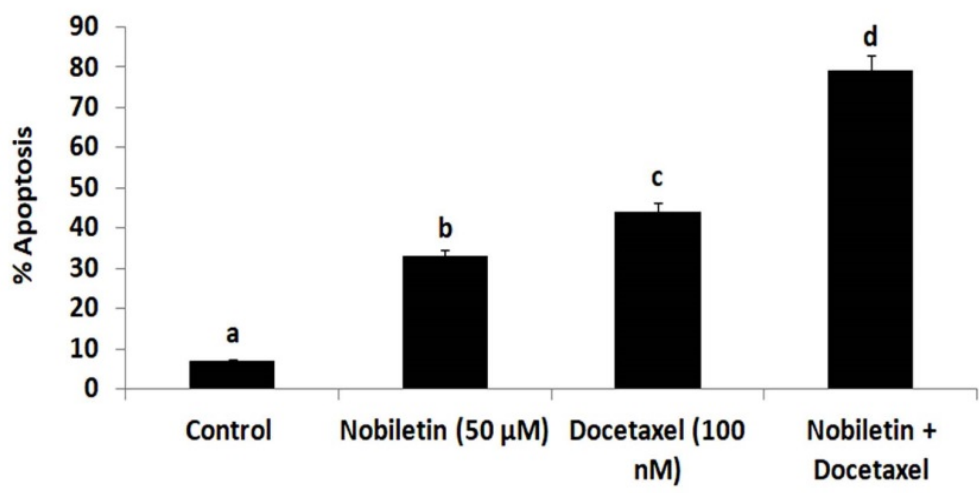

Fig. 2: The effect of nobiletin and docetaxel on intracellular ROS generation was evaluated with HCT-116 cells by using DCFH-DA staining; (A) Microscopic images (20 X) of non-treated HCT-116 and nobiletin with docetaxel treated HCT-116 cells. Untreated HCT-116 cells show weak fluorescence dicholoro fluorescent (DCF). Nobiletin with docetaxel treated HCT-116 cells shows increased ROS generation was designated into DCF fluorescence intensity; (B) Spectrofluorimetric analysis of ROS generation in HCT-116 cells. The statistical analysis was carried out using one way analysis of variance. Values are represented mean \pm standard deviation of three experiments. $\mathbf{p}<\mathbf{0 . 0 5}$ was significantly different from the control sample. 
A)

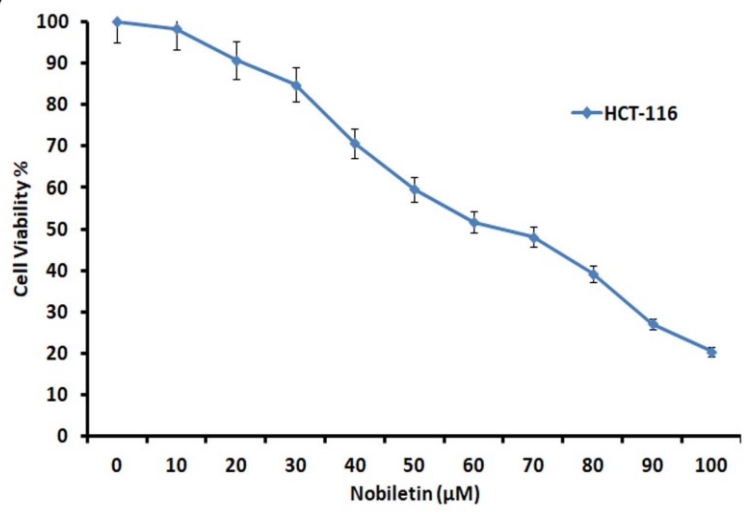

B)

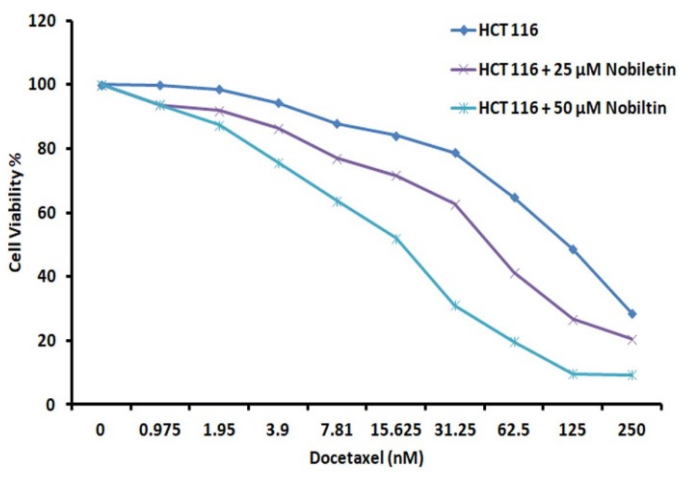

Fig. 3: The effect of nobiletin and docetaxel on mitochondria membrane potential damage was evaluated with HCT-116 cells using the Rhodamine 123; (A) Microscopic images (20 X) of nontreated HCT-116 and nobiletin with docetaxel treated HCT-116 cells. Untreated cells show high fluorescence indicate polarized mitochondria membrane. HCT-116 cells were treated with different concentration of nobiletin and docetaxel for $24 \mathrm{~h}$ and fluorescence intensity was decreased as indicate collapsed mitochondria matrix; (B) Spectrofluorimetric analysis of mitochondrion membrane potential in HCT-116 cells. The statistical analysis was carried out using one way analysis of variance. Values are represented mean \pm standard deviation of three experiments. $p<0.05$ was significantly different from the control sample.

nobiletin and docetaxel alone.

To further evaluate the action of nobiletin on docetaxelinduced apoptosis in the cells and the HCT-116 cells were treated with docetaxel and nobiletin. Then the cell apoptosis was measured by $\mathrm{EtBr} / \mathrm{AO}$ apoptotic morphological changes assay. Fig. 4, the microscopic picture exhibits the attribute features of apoptosis hallmark on nobiletin and docetaxel treated HCT-116 cells were stained with EtBr/AO. The EtBr red-coloured fluorescence dye was pierced into condensed nuclei in the apoptotic cells, whereas the AO (green) had uptake in live cells alone. Our findings illustrated control cells showed vastly green coloured fluorescence nucleus representing live cells (fig. 4). Nobiletin and docetaxel treated cells demonstrated orange colour, indicating that early-stage apoptosis and red coloured fragmented nuclei represent late apoptosis.

The control HCT-116 cells show increased phosphorylation of PI3K and AKT in HCT-116 cells. Moreover, we found that nobiletin sensitizes the cells and decreased fold of phosphorylated PI3K and AKT in HCT-116 cells (fig. 5). Interestingly, the combination of nobiletin and docetaxel treatment shows more effectively inhibits the PI3K and AKT expression in HCT-116.

The combination of nobiletin and docetaxel treatment regulated apoptotic factors in HCT-116 cells (fig. 6). This treatment more efficiently stimulated pro-apoptotic proteins include p53 and Bax protein expression than the nobiletin or docetaxel treatment alone. Furthermore, nobiletin combined with docetaxel treatment improves the over expression of cytochrome-c, caspase-3 and casepase-9 compared to nobiletin and docetaxel alone treated cells. Besides, nobiletin combined with docetaxel negatively regulates the Bcl-2 protein expression, thereby contributing to apoptosis in HCT116 cells.

Chemotherapy is a conventional anti-cancer therapy that strongly suppresses malignant tumours growth and development ${ }^{[19,20]}$. Previously, chemotherapeutic drugs are dispersed into cancerous tissue through microcirculation $^{[20-22]}$. Alternatively, the misdistribution of drugs probably leads to numerous defined

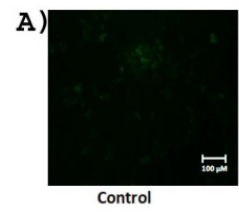

B)

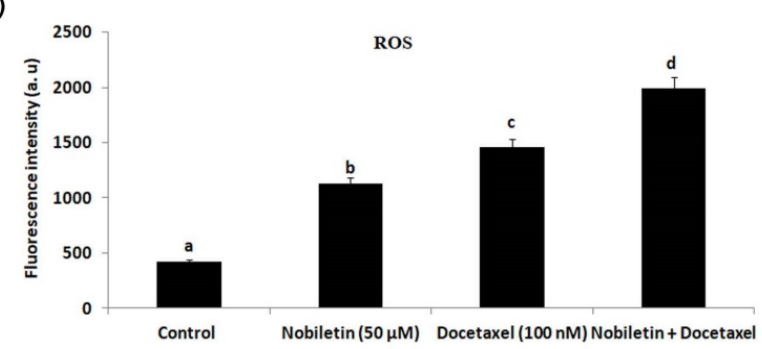

Fig. 4: Nobiletin combined docetaxel on apoptotic morphological changes in colon cancer cells HCT-116; (A) Microscopic images $(20 \mathrm{x})$ illustrated that apoptotic morphological changes conformed by dual staining (AO/EtBr); (B) Nobiletin and docetaxel treated HCT-116 cells shows increased \% of apoptotic cells than nobiletin and docetaxel alone. The statistical analysis was carried out using one way analysis of variance. Values are represented mean \pm standard deviation of three experiments. $\mathbf{p}<\mathbf{0 . 0 5}$ was significantly different from the control sample. 


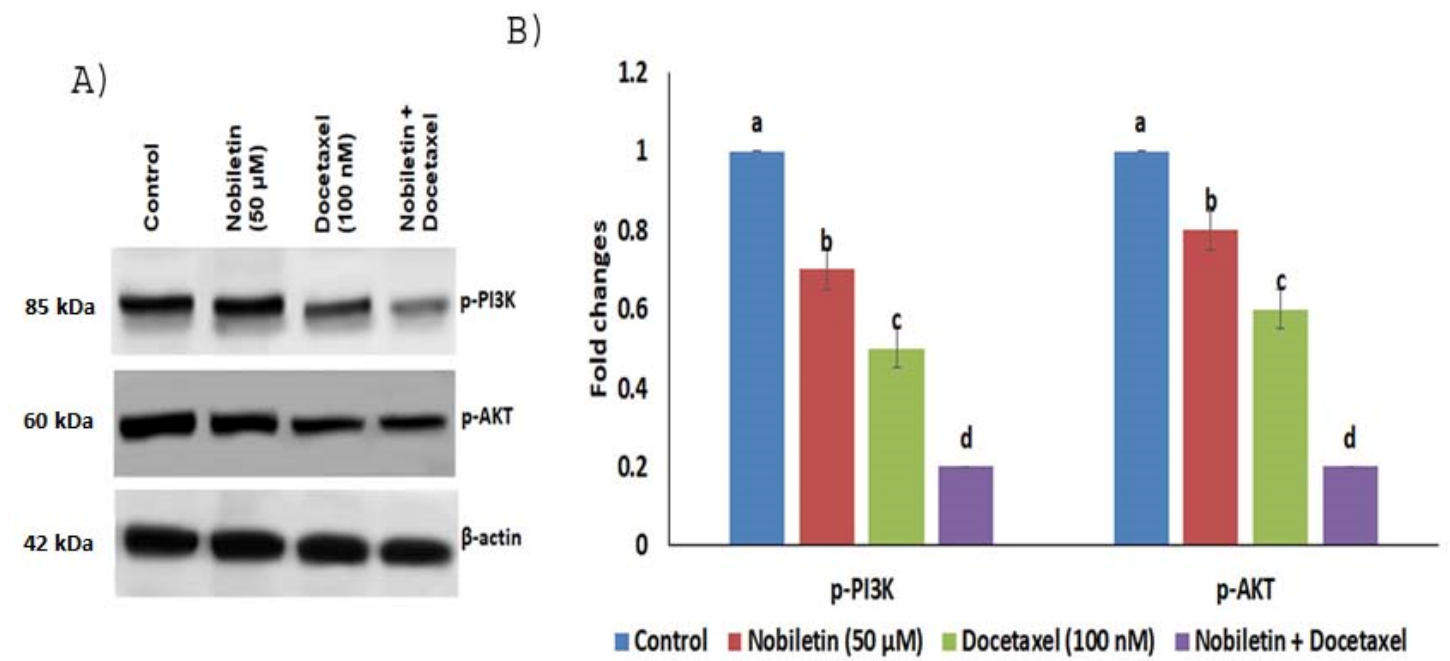

Fig. 5: Nobiletin combined docetaxel on PI3K/AKT signaling in colon cancer cells HCT-116; (A) Western blot experiments of p-PI3K and p-AKT expression in nobiletin combined docetaxel, nobiletin and docetaxel alone treated HCT-116 cells; (B) The densitometric data were accrued by Image $J$ software and obtained protein expressions were normalized by $B$-actin to ensure the equal loading of protein. The statistical analysis was carried out using one way analysis of variance. Values are represented mean \pm standard deviation of three experiments. $\mathbf{p}<0.05$ was significantly different from the control sample.

A)

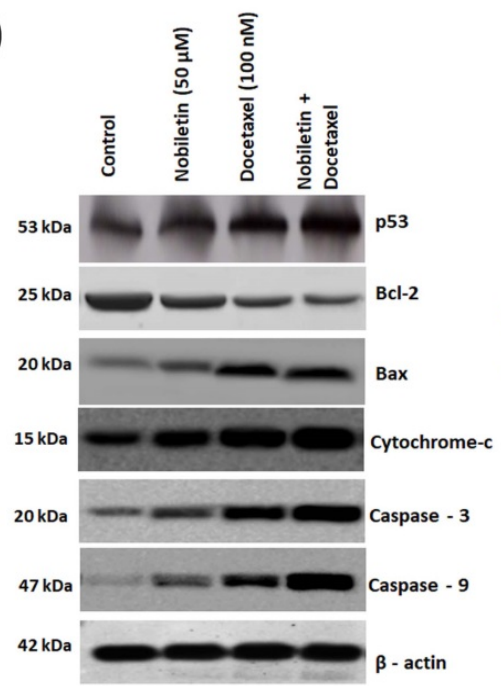

B)

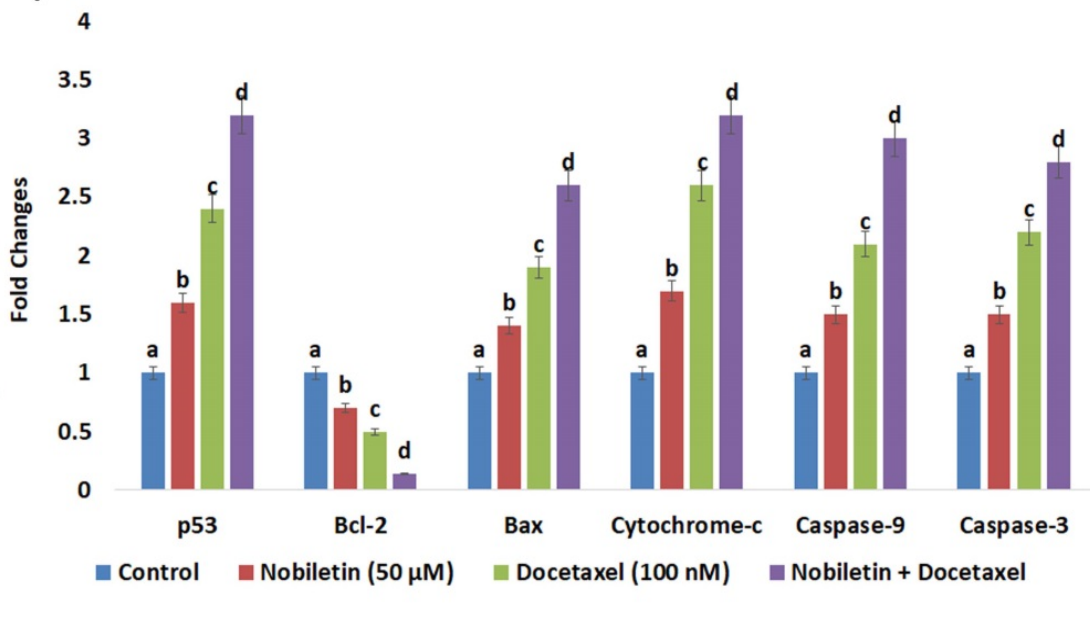

Fig. 6: Nobiletin combined docetaxel on apoptotic signaling in colon cancer cells HCT-116; (A) Western blot experiments of p53, Bcl-2, Bax, cytochrome-c, caspase-9 and caspase-3 expression in nobiletin combined docetaxel, nobiletin and docetaxel alone treated HCT-116 cells; (B) The densitometric data were accrued by Image $J$ software and obtained protein expressions were normalized by B-actin to ensure the equal loading of protein. The statistical analysis was carried out using one way analysis of variance. Values are represented mean \pm standard deviation of three experiments. $\mathbf{p}<\mathbf{0 . 0 5}$ was significantly different from the control sample.

modifications of tumors in the microenvironment, such as formation of hypoxic areas, elevation of interstitial fluid pressure and oligotrophy. These alterations decline the tumor's chemosensitivity and turn up into drug resistance ${ }^{[23]}$. Hence, improving the drug resistance of cancer cells has become a research focus. It has been investigated that Astragaloside IV sensitizes cancer cells to gefitinib by the regulation of Sirtuin 6 $\left(\right.$ SIRT6) ${ }^{[24]}$.

Nobiletin has been revealed to have anti-tumor properties in different cancer types, including breast cancer, lung cancer and gastric cancer, even though it's molecular mechanism remains unclear ${ }^{[25-27]}$. Furthermore, recent research illustrated that nobiletin sensitized cancer cells to chemotherapy ${ }^{[28,29]}$. For example, it stimulates apoptosis and then augments the anti-cancer drug's effects (5-fluorouracil) in human gastric cancer cells ${ }^{[30]}$. Nobiletin co-treatment and atorvastatin exhibit a strong synergy during the inhibition of colon carcinogenesis ${ }^{[31]}$. In this current investigation, we found that nobiletin improves the anti-cancer activities of docetaxel by this means enhances cell death in HCT-116 cells. 
Apoptosis is well-known to take part in a crucial role in a huge variety of biological process includes cell growth, replication, embryonic development, alterations in cell morphology and chemically mediated cell death. It is triggered through diverse stimuli, including an enhanced ROS level, DNA damage, activation of caspases family, cell shrinkage, chromatin condensation and nucleosome degradation ${ }^{[32]}$. Mitochondrial dysfunction and/or their loss of membrane potential induce the discharge of proapoptotic factors includes cytochrome $\mathrm{c}$ into the cytosol which in turn finally leads to activation of caspases cascades $^{[33]}$. Excessive ROS generation as well as accumulation impairs mitochondrial membranes in reverse, resultant into major MMP. This excessive generation of ROS and loss of MMP allowing the leakage of pro-apoptotic factors into the cytosolic $\operatorname{areas}^{[34]}$. Here in this research study we observed nobiletin and docetaxel co-treatment increased the ROS generation and as well damaged the MMP in HCT116 cells. Morphological characteristic of apoptosis is plasma membrane blebbing, loss of membrane integrity, ultrastructural modification of cytoplasmic organelles, condensation of chromatin, fragmentation of nucleus and the creation of apoptotic bodies. Several chemotherapeutic agents target cancer cell and ability to stimulate the cell death via the formation of apoptotic bodies $^{[35]}$. Additionally, we also observed that cellular morphological alterations and cell death induced by nobiletin and docetaxel in HCT-116 cells.

Anomalous activation of PI3K/AKT signaling pathway was common in human cancer models ${ }^{[36]}$. Several drugs targeting nodes of this pathways have been targeted due to inducing apoptosis ${ }^{[37]}$. Moreover, Activated AKT and it signaling involved playing an anti-apoptotic role by phosphorylation of pro-apoptotic mediators ${ }^{[38]}$. In our study, combined delivery of nobiletin and docetaxel significantly decreased the phosphorylation levels of PI3K and AKT protein expression that has been confirmed by Western blotting. These results designated that combined delivery of nobiletin and docetaxel affected the HCT-116 cell survival inhibiting both PI3K-AKT pathways. The tumors arise not because of growth stimulation but due to the inhibition of cell death by Bcl-2, thereby it leads to the accumulation of cells ${ }^{[39]}$. The interaction between Bcl-2 and Bax protein by which in turn simulates apoptosis, the proportion of Bcl-2: Bax comparatively than the whole quantity is thought to be the essential quantity of cell survival rate ${ }^{[40]}$. Our current finding results exhibited that nobiletin and docetaxel treatment positively regulated the Bax expression and negatively regulated the Bcl-
2 protein expression in HCT-116 cells. In addition, we found that nobiletin and docetaxel considerably stimulates the caspase protein expression in HCT116 cells. Tumor-suppressor protein p53 proteins critically playing an important role for the induction of apoptosis $^{[41]}$. It may regulate apoptosis through its capability to stimulate the transcription process of Bax gene and also to inhibit the transcription of Bcl-2. Conversely, p53 also stimulate cell death by means of transcription independent mechanism ${ }^{[42]}$. In this current investigation, we observed that nobiletin and docetaxel improved the p53 expression pattern in HCT-116 cells.

In this present work, we concluded that nobiletin combined docetaxel treatment increased the cytotoxicity through increased the ROS generation in HCT-116. The combined treatment of nobiletin and docetaxel increased the alteration mitochondrion membrane mediated apoptotic signaling in HCT-116 cells. Hence, this study proved that nobiletin may act as chemosensitive efficacy and also afford innovative insight into the mixture of nobiletin and chemotherapeutic drugs in treatment colorectal cancers.

\section{Conflict of interest:}

All the authors stating that there are no conflicts of interest.

\section{REFERENCES}

1. Armaghany T, Wilson JD, Chu Q, Mills G. Genetic alterations in colorectal cancer. Gastrointest Cancer Res 2012;5(1):19.

2. Carethers JM, Jung BH. Genetics and genetic biomarkers in sporadic colorectal cancer. Gastroenterology 2015;149(5):1177-90.

3. Alsanea N. The dilemma of the threshold age to start screening for colorectal cancer in Saudi Arabia. Saudi J Gastroenterol 2014;20(3):141-2.

4. Alfarouk KO, Stock CM, Taylor S, Walsh M, Muddathir $\mathrm{AK}$, Verduzco D, et al. Resistance to cancer chemotherapy: failure in drug response from ADME to P-gp. Cancer Cell Int 2015;15(1):71.

5. Masui K, Gini B, Wykosky J, Zanca C, Mischel PS, Furnari FB, et al. A tale of two approaches: complementary mechanisms of cytotoxic and targeted therapy resistance may inform nextgeneration cancer treatments. Carcinogenesis 2013;34(4):72538 .

6. Gerhards NM, Rottenberg S. New tools for old drugs: functional genetic screens to optimize current chemotherapy. Drug Resist Updat 2018;36:30-46.

7. Hassan M, Watari H, AbuAlmaaty A, Ohba Y, Sakuragi N. Apoptosis and molecular targeting therapy in cancer. BioMed Res Int 2014;2014:150845.

8. Pfeffer CM, Singh AT. Apoptosis: a target for anticancer therapy. Int J Mol Sci 2018;19(2):448.

9. Demain AL, Vaishnav P. Natural products for cancer chemotherapy. Microb Biotechnol 2011;4:687-99.

10. Coventry BJ, Ashdown ML. Complete clinical responses 
to cancer therapy caused by multiple divergent approaches: a repeating theme lost in translation. Cancer Manag Res 2012;4:137.

11. van der Veldt AA, Lubberink M, Mathijssen RH, Loos WJ, Herder GJ, Greuter HN, et al. Toward prediction of efficacy of chemotherapy: a proof of concept study in lung cancer patients using [11C] docetaxel and positron emission tomography. Clin Cancer Res 2013;19(15):4163-73.

12. Wu YQ, Zhou CH, Tao J, Li SN. Antagonistic effects of nobiletin, a polymethoxyflavonoid, on eosinophilic airway inflammation of asthmatic rats and relevant mechanisms. Life Sci 2006;78(23):2689-96.

13. Kunimasa K, Kuranuki S, Matsuura N, Iwasaki N, Ikeda M, Ito A, et al. Identification of nobiletin, a polymethoxyflavonoid, as an enhancer of adiponectin secretion. Bioorg Med Chem Lett 2009;19(7):2062-4.

14. Ye L, Yuan G, Xu F, Sun Y, Chen Z, Chen M, et al. The smallmolecule compound BM-1197 inhibits the antiapoptotic regulators $\mathrm{Bcl}-2 / \mathrm{Bcl}-\mathrm{xL}$ and triggers apoptotic cell death in human colorectal cancer cells. Tumor Biol 2015;36(5):344755.

15. NilamberLal Das R, Muruhan S, Nagarajan RP, Balupillai A. Naringin prevents ultraviolet- $B$ radiation-induced oxidative damage and inflammation through activation of peroxisome proliferator-activated receptor $\gamma$ in mouse embryonic fibroblast (NIH-3T3) cells. J Biochem Mol Toxicol 2019;33(3):e22263.

16. Sabitha R, Nishi K, Gunasekaran VP, Agilan B, David E, Annamalai G, et al. p-Coumaric acid attenuates alcohol exposed hepatic injury through MAPKs, apoptosis and Nrf2 signaling in experimental models. Chem Biol Interact 2020;321:109044.

17. Baskić D, Popović S, Ristić P, Arsenijević NN. Analysis of cycloheximide-induced apoptosis in human leukocytes: Fluorescence microscopy using annexin V/propidium iodide versus acridin orange/ethidium bromide. Cell Biol Int 2006;30(11):924-32.

18. Lowry $\mathrm{OH}$, Rosebrough NJ, Farr AL, Randall RJ. Protein measurement with the Folin phenol reagent. J Biol Chem 1951;193:265-75.

19. Thorpe LM, Yuzugullu H, Zhao JJ. PI3K in cancer: divergent roles of isoforms, modes of activation and therapeutic targeting. Nat Rev Cancer 2015;15(1):7-24.

20. Choi IK, Strauss R, Richter M, Yun CO, Lieber A. Strategies to increase drug penetration in solid tumors. Front Oncol 2013;3:193.

21. Stylianopoulos T, Jain RK. Combining two strategies to improve perfusion and drug delivery in solid tumors. Proc Natl Acad Sci USA 2013;110(46):18632-7.

22. Forster JC, Harriss-Phillips WM, Douglass MJ, Bezak E. A review of the development of tumor vasculature and its effects on the tumor microenvironment. Hypoxia 2017;5:21.

23. Yeldag G, Rice A, del Río Hernández A. Chemoresistance and the self-maintaining tumor microenvironment. Cancers 2018;10(12):471.

24. Dai PC, Liu DL, Zhang L, Ye J, Wang Q, Zhang HW, et al. Astragaloside IV sensitizes non-small cell lung cancer cells to gefitinib potentially via regulation of SIRT6. Tumor Biol 2017;39(4):1010428317697555.

25. Chen C, Ono M, Takeshima M, Nakano S. Antiproliferative and apoptosis-inducing activity of nobiletin against three subtypes of human breast cancer cell lines. Anticancer Res 2014;34(4):1785-92.

26. Da C, Liu Y, Zhan Y, Liu KA, Wang R. Nobiletin inhibits epithelial-mesenchymal transition of human non-small cell lung cancer cells by antagonizing the TGF- $\beta 1 / \mathrm{Smad} 3$ signaling pathway. Oncology Rep 2016;35(5):2767-74.

27. Yoshimizu N, Otani Y, Saikawa Y, Kubota T, Yoshida M, Furukawa T, et al. Anti-tumour effects of nobiletin, a citrus flavonoid, on gastric cancer include: Antiproliferative effects, induction of apoptosis and cell cycle deregulation. Aliment Pharmacol Ther 2004;20:95-101.

28. Ma W, Feng S, Yao X, Yuan Z, Liu L, Xie Y. Nobiletin enhances the efficacy of chemotherapeutic agents in ABCB1 overexpression cancer cells. Sci Rep 2015;5(1):18789.

29. Li N, Zhang Z, Jiang G, Sun H, Yu D. Nobiletin sensitizes colorectal cancer cells to oxaliplatin by PI3K/Akt/MTOR pathway. Front Biosci 2019;24:303-12.

30. Moon JY, Cho M, Ahn KS, Cho SK. Nobiletin induces apoptosis and potentiates the effects of the anticancer drug 5-fluorouracil in p53-mutated SNU-16 human gastric cancer cells. Nutr Cancer 2013;65(2):286-95.

31. Wu X, Song M, Qiu P, Rakariyatham K, Li F, Gao Z, et al. Synergistic chemopreventive effects of nobiletin and atorvastatin on colon carcinogenesis. Carcinogenesis 2017;38(4):455-64.

32. Elmore S. Apoptosis: a review of programmed cell death. Toxicol Pathol 2007;35(4):495-516.

33. Gupta S, Kass GE, Szegezdi E, Joseph B. The mitochondrial death pathway: a promising therapeutic target in diseases. $\mathrm{J}$ Cell Mol Med 2009;13(6):1004-33.

34. Wang L, Yeung JH, Hu T, Lee WY, Lu L, Zhang L, et al. Dihydrotanshinone induces p53-independent but ROS-dependent apoptosis in colon cancer cells. Life Sci 2013;93(8):344-51.

35. Muthusamy G, Balupillai A, Ramasamy K, Shanmugam M, Gunaseelan S, Mary B, et al. Ferulic acid reverses ABCB1mediated paclitaxel resistance in MDR cell lines. Eur J Pharmacol 2016;786:194-203.

36. Shi X, Wang J, Lei Y, Cong C, Tan D, Zhou X. Research progress on the PI3K/AKT signaling pathway in gynecological cancer. Mol Med Rep 2019;19(6):4529-35.

37. Khan M, Maryam A, Qazi JI, Ma T. Targeting apoptosis and multiple signaling pathways with icariside II in cancer cells. Int J Biol Sci 2015;11(9):1100.

38. Zhong D, Liu X, Khuri FR, Sun SY, Vertino PM, Zhou W. LKB1 is necessary for Akt-mediated phosphorylation of proapoptotic proteins. Cancer Res 2008;68(18):7270-7.

39. Kelly PN, Strasser A. The role of Bcl-2 and its pro-survival relatives in tumourigenesis and cancer therapy. Cell Death Differ 2011;18(9):1414-24.

40. Placzek WJ, Wei J, Kitada S, Zhai D, Reed JC, Pellecchia M. A survey of the anti-apoptotic Bcl-2 subfamily expression in cancer types provides a platform to predict the efficacy of Bcl2 antagonists in cancer therapy. Cell Death Dis 2010;1(5):e40.

41. Ozaki T, Nakagawara A. Role of $\mathrm{p} 53$ in cell death and human cancers. Cancers 2011;3(1):994-1013.

42. Vaseva AV, Moll UM. The mitochondrial p53 pathway. Biochim Biophys Acta 2009;1787(5):414-20. 